nature

immunology

\title{
Hyping research
}

\section{Reporting of scientific research is sometimes exaggerated or at worse inaccurate. Researchers need to change this and have the power to do so.}

6 4 Man flu is not a myth: Female hormones give women stronger immune systems," declares the Daily Mail. "Burger fellas firing blanks," says the Sun. Drinking red wine is beneficial because of its "cancer-busting antioxidant," hails the Daily Express. More recently, with much fanfare, we were introduced to Ida, a 47-million-year-old fossil of a lemur-like animal with human-like hands that was hailed by the press as a "missing link" in human evolution that will rewrite textbooks. And so the sensationalist headlines go on.

Although these attention-grabbing headlines might help sell papers and increase traffic to newspaper websites, such reporting is irresponsible to the public and to science in general. Even if the article itself is more balanced, it must be remembered that many readers never get much beyond the headline. The net result is the public comes away with much misinformation. In the 'man flu' story, for example, the Daily Mail failed to report that the research this story was based on did not investigate infection with influenza, used mouse models and cannot prove whether 'man flu' exists or not. The articles reporting an association of steak, burgers and full-fat cream with poor-quality sperm failed to state that this was a small case-control study that did not prove causation and that even the authors of the study were cautious in the conclusions that can be drawn from this research.

Somewhat more disturbing is the hype surrounding Ida. This paper was marketed in an unusual manner. The unveiling of the fossil-aptly named Darwinius masillae - preceded publication of the paper in PLoS $O N E$ with a cleverly orchestrated event at the American Museum of Natural History in New York hosted by Mayor Michael Bloomberg. A book (The Link), two documentaries, a website and even a Twitter feed had also been prepared before publication.

In addition, Jørn Hurum, the senior author of the study, described Ida to the public as "the Holy Grail for paleontologists" and as the first link to all humans. Although communicating scientific discovery to the public is admirable, this choice of words was perhaps inaccurate. To call Ida the 'missing link' in human evolution will require more research, and at present the scientific community as a whole is rather skeptical. Notably, in the paper itself, the authors are more cautious and never call Ida the missing link. Sadly, the press did not relay these facts.

Why is science so often sensationalized by the media? The state of scientific reporting, at least mainstream reporting, is in a state of disarray. Newsrooms across the globe are shedding full-time specialty scientific reporters, with the latest casualty being The Boston Globe and $\mathrm{CNN}$. Increasingly, science stories are being covered by nonscientists with little or no knowledge of the intricacies that make solid research. Attention-grabbing headlines, rather than solid reporting or in-depth analysis, are becoming more prevalent. More often than not, reporters rely heavily on press releases, often regurgitating them as fact without doing any investigative journalism.

At a media panel at the recent annual meeting of the National Association of Science Writers, Charles Petit, a veteran science reporter and media critic, raised this issue by claiming that cleverly written press releases are converting so-so research into must-read stories. Petit cited an example in which University of Utah research about the mating habits of primitive cycad plants, which could be considered far from sexy, was released to the press with the title "Living fossils have hot sex." The press release was subsequently copied and translated as "Primitive plants have hot, stinky sex" by Reuters. Clearly, it is imperative that press releases are not passed off as news stories.

In addition, the changing face of scientific journalism is being propelled by the online revolution. Newspapers and traditional forms of media are struggling to compete with online news outlets, including blogs. Although some blogs written by informed scientists are sources of balanced and insightful information, the internet being as it is means that anyone can put news on the web and perpetuate pseudo-science. Confounding this problem is the fact that a person's political and religious beliefs often dictate where on the web they seek their information. The resulting combination of unreliable reporting and biased reading can be disastrous.

Although this paints a rather pessimistic picture of scientific journalism, there is cause for optimism. Several websites that critique the scientific press have started to evolve. In the UK, the National Health Service provides an unbiased and evidence-based analysis of health stories that hit the media (http://www.nhs.uk/Pages/HomePage.aspx). HealthNewsReview.org is a website dedicated to improving the accuracy of news stories about medical treatments, tests, products and procedures. The Yale Environment 360 website provides unbiased opinions from scientists and journalists on issues such as climate change. These are just a handful of examples.

Organizations such as the Voice of Young Science network are providing advice for researchers on how to stand up for science in public and correct misinformation in the mass media. Although scientists should be encouraged to discuss research with reporters, they need to carefully prepare for such interviews much as they do for presentations at scientific conferences. They need to resist a journalist's efforts to direct the interview to the long-term applications of the research, which then often become the main theme of the story. Scientists need to let editors know if a reporter exaggerates or is inaccurate in the final story. Posting comments on articles and petitioning political powers are other ways researchers can set the record straight.

As George Bernard Shaw once said, "The single biggest problem in communication is the illusion that it has taken place." Scientists need to ensure this is not an illusion but a reality. 